$\mathrm{T}$ concentration to be associated with more severe strokes, as assessed by the Scandinavian stroke scale. However, we could not correlate computed tomography data with clinical outcomes. Computed tomography was usually performed in the first 24 hours to differentiate between ischaemic and haemorrhagic strokes. In the first 48 hours, however, the extent of an acute infarct is often poorly defined on computed tomography. A previous study found that infarct volume measured by computed tomography was poorly correlated with clinical measures of stroke severity and outcome. $^{14}$

Troponin $\mathrm{T}$ concentration was measured in a single serum sample obtained 12 to 72 hours after admission to hospital. Serial measurement of troponin T concentration and creatine kinase $\mathrm{MB}$ over the following few days would be needed to determine the time course of myocardial damage after an ischaemic stroke.

Contributors: CJE initiated the study and contributed to the study design, analysis of the data, interpretation, and reporting. PJ collected the data and contributed to the study design, interpretation, and reporting. RMLW analysed the data and contributed to interpretation and reporting. JH helped to initiate the study and, with ARM and NEA, contributed to interpretation and reporting. All authors are guarantors.

Funding: None.
Competing interests: None declared.

1 Bonita R, Beaglehole R. Cerebrovascular disease: explaining stroke mortality trends. Lancet 1993:341:1510-1.

2 Anderson NE, Bonita R, Broad JB. Early management and outcome of acute stroke in Auckland. Aust N Z Med J 1997;27:561-7.

3 Brickner ME. Cardioembolic stroke. Am J Med 1996;100:465-74.

4 Samuels MA. Neurally induced cardiac damage: definition of the problem. Neurol Clin 1993;11:273-92.

5 Norris JW, Hachinski VC, Myers MG, Callow J, Wong T, Moore RW. Serum cardiac enzymes in stroke. Stroke 1979;10:548-53.

6 Donnelly R, Millar-Craig MW. Cardiac troponins: IT upgrade for the heart. Lancet 1998;351:537-9.

7 Scandinavian Stroke Study Group. Multicenter trial of hemodilution in ischemic stroke: background and study protocol. Stroke 1985;16:885-90.

8 Reith J, Jorgensen HS, Pedersen PM, Nakayama H, Raaschow HO, Jeppersen LL, et al. Body temperature in acute strokes: relation to stroke severity, infarct size, mortality and outcome. Lancet 1996;347:422-5.

9 Weir JW, Gordon DM, Alexander GD, Kennedy RL. Is hypoglycaemia an independent predictor of poor outcome after acute stroke? Results of a long term follow up study. BMJ 1997;314:1303-6.

10 Christenson RH, Duh SH, Newby LK, Ohman EM, Califf RM, Granger $\mathrm{CB}$, et al. Cardiac troponin $\mathrm{T}$ and cardiac troponin I: relative values in short term risk stratification of patients with acute coronary syndromes. Clin Chem 1998;44:494-501.

11 McNeil AR, Marshall M, Ellis CJ, Hawkins RC. Why is troponin T increased in the serum of patients with end-stage renal disease? Clin Chem 1998:44:2377-8.

12 Myers MG, Norris JW, Hachinski VC, Weingert ME, Sole MJ. Cardiac sequelae of acute stroke. Stroke 1982;13:838-42.

13 Myers MG, Norris JW, Hachinski VC, Sole MJ. Plasma norepinephrine in stroke. Stroke 1981;12:200-4.

14 Saver JL, Johnston KC, Homer D, Wityk R, Koroshetz W, Truskowski LL, et al. Infarct volume as a surrogate or auxiliary outcome measure in ischemic stroke clinical trials. Stroke 1999;30:293-8.

(Accepted 22 February 2000)

\title{
Single blind, randomised trial of efficacy and acceptability of oral Picolax versus self administered phosphate enema in bowel preparation for flexible sigmoidoscopy screening
}

Wendy S Atkin, Andrew Hart, Robert Edwards, Claire F Cook, Jane Wardle, Peter McIntyre, Roger Aubrey, Clare Baron, Stephen Sutton, Jack Cuzick, Asha Senapati,John M A Northover

Imperial Cancer Research Fund Colorectal Cancer Unit, St Mark's Hospital, Northwick Park, Harrow

HA1 3UJ

Wendy S Atkin deputy director

Claire F Cook

statistician

Clare Baron

data manager

John M A

Northover

director

Leicester General Hospital, Leicester LE5 4PW

Andrew Hart senior registrar in gastroenterology continued over

\begin{abstract}
Objective To compare the acceptability and efficacy of two methods of self administered bowel preparation for flexible sigmoidoscopy screening: a single phosphate enema and a single sachet of Picolax. Design Single blind, randomised trial. Setting Endoscopy units of two general hospitals. Participants 1442 men and women aged 55-64 years who had agreed to be screened by flexible sigmoidoscopy.

Main outcome measures Attendance rates, compliance with allocated preparations, adverse effects, quality of bowel preparation, procedure time, and yield of neoplasia.

Results Compliance with the enema was higher than with the Picolax (608 (84\%) v 566 (79\%); difference $6 \%, 95 \%$ confidence interval $2 \%$ to $10 \%$ ). Almost half of those who refused Picolax used an enema at home. Wind, incontinence, and sleep disturbance were more frequent in the Picolax group than the enema group; bottom soreness was more frequent in the enema group. Around 30\% (187) found the diet restriction required by Picolax difficult; 78\% (471) found the enema easy to administer. The quality of preparation
\end{abstract}

was better with the enema; the proportion of procedures complete to the descending colon was greater and the mean duration of the procedure was shorter. There was no significant difference in polyp detection rates.

Conclusion A single phosphate enema self administered around one hour before leaving home is a more acceptable and effective method of preparing the distal bowel for flexible sigmoidoscopy than Picolax.

\section{Introduction}

A randomised trial is in progress in the United Kingdom to examine the efficacy of a single flexible sigmoidoscopy screen in the prevention of bowel cancer. ${ }^{1}$ At the start of the trial we wished to identify the most acceptable and effective method of bowel preparation, and we considered an enema or an oral laxative. Enemas are the preferred option since they quickly clear the lower bowel and require no dietary restriction, whereas oral preparations require a liquid diet for at least 12 hours before the procedure. ${ }^{2}$

In the United Kingdom the enema is generally administered by a nurse in the unit, but the trial did not 
have the staff or toilet facilities. In the United States, the enema is often self administered at home, ${ }^{3}$ but the willingness or ability of asymptomatic British people to self administer an enema was not known, and we suspected that an oral laxative might be preferred.

We compared a single self administered phosphate enema (Fletchers' phosphate enema (long tube version for self administration), Pharmax, Bexley, Kent) with a single sachet of oral sodium picosulphate with magnesium citrate (Picolax). Two sachets of Picolax are used routinely for colonoscopy, but a single sachet is used for flexible sigmoidoscopy. ${ }^{4}$ A single phosphate enema is as effective as two enemas for sigmoidoscopy. ${ }^{35}$ The two preparations were compared in two separate populations to determine whether the findings could be generalised throughout the United Kingdom.

\section{Participants and methods}

\section{Study design}

The study was carried out in Welwyn Garden City in 1995 and Leicester in 1996 as part of the pilot studies for the UK flexible sigmoidoscopy screening trial. Approval for the studies was obtained from the hospitals' ethics committees. Men and women aged 55-64 who met the eligibility criteria ${ }^{6}$ and were randomised to an invitation for screening were further randomised to receive oral laxative or an enema after they had confirmed their appointment. Clear, detailed instructions were included, and a nurse was available for telephone advice if required. Participants were advised to use the enema about 1 hour before leaving home for the screening examination and not to eat after using the enema. Participants who were sent Picolax were advised to take it at either 2 pm or $6 \mathrm{pm}$ on the day before screening for a morning or afternoon examination respectively and to have no solid food thereafter until after the examination.

Participants who cancelled their appointment after receiving their bowel preparation were offered an alternative. Those sent the enema could have the enema in the unit or Picolax, and those sent Picolax could have an enema at home or in the unit.

The endoscopists were unaware of the preparation used. After passing each $15 \mathrm{~cm}$ section of the bowel, they noted the percentage of mucosa area visible. At the end of the examination, the endoscopists rated the adequacy of the bowel preparation as excellent, good, adequate, or poor. ${ }^{7}$ If the preparation was poor, sigmoidoscopy was repeated after administration of an enema in the unit.

Participants were asked to complete a questionnaire in the unit just before screening. This asked about usual bowel habit, problems in the use of the allocated preparation, the severity of an itemised list of adverse effects, and whether they would be prepared to use the same preparation again. They were given an additional questionnaire to complete on the following morning asking about problems experienced during or after the procedure.

\section{Randomisation}

Block randomisation to Picolax or enema was performed centrally. Administrative staff at trial centres were responsible for posting bowel preparations. Both administrative staff and participants were asked not to disclose their method of bowel preparation to the endo- scopist. Participants had given consent to take part in the screening trial, but we did not ask for separate consent for randomisation to different bowel preparation methods. This decision was made because both preparations are in routine use, the side effects are minor, and in the absence of this study all participants would have been allocated one of the preparations.

\section{Statistical methods}

Sample size calculations for the acceptability study were based on $85 \%$ compliance in the Picolax group and $75 \%$ in the enema group. For the efficacy study, calculations were based on good or excellent bowel preparation in $70 \%$ of subjects in the Picolax group ${ }^{4}$ and $85 \%$ in the enema group. ${ }^{8}$ We estimated that we needed a population of 1100 to achieve $98 \%$ power for the overall comparisons of the two treatment groups (with at least $75 \%$ power within each centre).

We calculated absolute risk differences (with exact $95 \%$ confidence intervals) to compare proportions in the enema and Picolax groups. Duration of examinations and visibility in each group were compared by $t$ tests. Differences between centres were assessed by the Mantel-Haenszel test for homogeneity of odds ratios.

\section{Results}

We sent Picolax to 721 people (Welwyn Garden City: 299; Leicester: 422) and an enema to 721 (Welwyn Garden City: 290; Leicester: 431). The groups were well matched at entry. The average age was 61 years in both groups in Welwyn Garden City and 60 years in Leicester, and the proportion of men in the groups was similar in each centre (Welwyn Garden City: 45\% (267); Leicester: $49 \%$ (421)).

\section{Compliance}

Compliance with the enema was higher than with Picolax (84\% v 79\%; difference 5\%, 95\% confidence interval $2 \%$ to $10 \%$ ) (table 1). This effect was seen in both centres. There was no significant difference between the enema and Picolax groups in the proportions who used an alternative bowel preparation (3\% in enema group $v 4 \%$ Picolax). Of the 63 participants sent Picolax who requested an enema instead, 27 (43\%) used it at home. By contrast, only $18(27 \%)$ of the 67 who refused to use the enema at home asked to use the oral preparation; the remainder requested the enema in the unit. Only $49(7 \%)$ participants sent the enema requested to have it in the unit.

\section{Acceptability}

Of those who used the allocated bowel preparation, about $90 \%$ of each group in each centre reported they would be willing to use the same preparation again (table 2). Most of those using the enema at home (471, $78 \%)$ found it easy to administer; $11(2 \%)$ found it very difficult or needed it to be administered by someone else. Five hundred and forty three $(96 \%)$ of those sent Picolax took the full sachet, but a third (187) found it difficult to cope with no food and $45(8 \%)$ found it very difficult or had to have something to eat.

A higher proportion felt unwell after taking the enema than after the Picolax (88 (15\%) v 39 (7\%), 95\% confidence interval for difference $4 \%$ to $11 \%$ ), but over $80 \%$ of both groups felt quite normal. More of the
Imperial Cancer Research Fund Department of Mathematics, Statistics and Epidemiology, Lincoln's Inn Fields, London

WC2A 3PX

Robert Edwards statistician

Jack Cuzick

head

Imperial Cancer Research Fund Health Behaviour Unit, Department of Epidemiology and Public Health, University College, London W1E 6BT

Jane Wardle professor

Stephen Sutton reader in social and health psychology

Queen Elizabeth II Hospital, Welwyn Garden City

AL7 4HQ

Peter McIntyre consultant gastroenterologist Roger Aubrey hospital practitioner Queen Alexandra Hospital, Portsmouth Asha Senapati consultant surgeon

Correspondence to: W Atkin atkin@icrf.icnet.uk 
Table 1 Attendance and use of allocated bowel preparation. Values are numbers (percentages) of participants

\begin{tabular}{|c|c|c|c|c|c|c|c|c|}
\hline & \multicolumn{3}{|c|}{ Total } & \multicolumn{2}{|c|}{ Welwyn Garden City } & \multicolumn{2}{|c|}{ Leicester } & \multirow[b]{2}{*}{ P value } \\
\hline & Enema & Picolax & $\begin{array}{c}\text { Difference in } \%(95 \% \\
\text { CI) }\end{array}$ & Enema & Picolax & Enema & Picolax & \\
\hline Sent bowel preparation & 721 & 721 & & 290 & 299 & 431 & 422 & \\
\hline Attended and used allocated preparation at home & $608(84)$ & $566(79)$ & $5(2$ to 10$)$ & $248 \dagger(86)$ & $237(79)$ & $360(84)$ & $329(78)$ & 0.79 \\
\hline Attended but refused to use allocated preparation at home: & $67(9)$ & $63(9)$ & $0(-2$ to 4$)$ & $23(8)$ & $28(9)$ & $44(10)$ & $35(8)$ & 0.28 \\
\hline Used alternative preparation at home & $18(2)$ & $27(4)$ & $-1(-3$ to 1$)$ & $4(1)$ & $12(4)$ & $14(3)$ & $15(4)$ & 0.15 \\
\hline
\end{tabular}

* Significance test for homogeneity of bowel preparation effect between Welwyn Garden City and Leicester

tOne subject did not complete a bowel preparation questionnaire and is therefore not included in analyses of acceptability and efficacy.

itemised adverse effects were rated as moderate or severe in the Picolax groups, including wind, incontinence, and sleep disturbance, with two people complaining that the Picolax began to work in the middle of the night producing incontinence in bed. Of the adverse effects rated as moderate or severe in the enema group, only bottom soreness was reported more frequently than for Picolax.

In all, 94\% (925/984) participants completed a further questionnaire on the morning after the test. Bottom soreness was the only adverse effect reported significantly more frequently in the enema group (table 3). This effect was seen in both centres. Rates of wind, incontinence, and sleep disturbance were not higher in the Picolax group on the morning after the test. There were no differences between the preparations in the pain experienced during the test.

Efficacy

On several measures, the enema performed better than the Picolax (table 4). The quality of preparation was assessed by endoscopists as excellent or good in a significantly higher proportion of participants using the enema in both centres. The mean visibility was also better with the enema in the first $45 \mathrm{~cm}$ from the anus in both centres. The proportion of examinations reaching the descending colon or beyond was significantly higher and the duration of the examination was significantly shorter in the enema group in both centres. Despite the apparent better performance of the enema, the proportion of people in whom polyps, adenomas, or cancers were detected and the total number of polyps detected overall did not differ materially between the preparations.

\section{Discussion}

Bowel preparation for screening flexible sigmoidoscopy needs to be quick and easy and to cause the minimum of discomfort in order to increase compliance. It should ideally be undertaken at home to reduce the workload of nursing staff and the congestion in the endoscopy unit if a large number of people are screened at each session. Effective bowel preparation is essential to increase the chance of detection of neoplasms (particularly if the examination is to be undertaken only once, as we have proposed $^{1}$ ); to aid insertion of the endoscope to its maximal point; to reduce the time required for the examination; to reduce patient discomfort during endoscopy; and to reduce the need for repeat examinations, which would increase procedure costs and inconvenience.

Table 2 Acceptability of Picolax and self administered enema as reported on an itemised questionnaire completed before screening. Values are numbers (percentages) of participants

\begin{tabular}{|c|c|c|c|c|c|c|c|c|}
\hline & \multicolumn{3}{|c|}{ Total } & \multicolumn{2}{|c|}{ Welwyn Garden City } & \multicolumn{2}{|c|}{ Leicester } & \multirow[b]{2}{*}{ P value * } \\
\hline & Enema & Picolax & Difference in \% $(95 \% \mathrm{Cl})$ & Enema & Picolax & Enema & Picolax & \\
\hline Total using allocated preparation & 607 & 566 & & 247 & 237 & 360 & 329 & \\
\hline \multicolumn{9}{|l|}{ Ease of administration of enema: } \\
\hline Easy & $471(78)$ & NA & & $188(76)$ & NA & $283(79)$ & NA & \\
\hline Quite difficult & $91(15)$ & NA & & $50(20)$ & NA & $41(11)$ & NA & \\
\hline Very difficult & $7(1)$ & NA & & $4(2)$ & NA & $3(1)$ & NA & \\
\hline Given by another person & $4(1)$ & NA & & $4(2)$ & NA & 0 & NA & \\
\hline \multicolumn{9}{|l|}{ Coping with no food: } \\
\hline No problem & NA & $356(63)$ & & NA & $160(67)$ & NA & $196(60)$ & \\
\hline A bit difficult & NA & $142(25)$ & & NA & $53(22)$ & NA & $89(27)$ & \\
\hline Very difficult & NA & $43(8)$ & & NA & $19(8)$ & NA & $24(7)$ & \\
\hline Couldn't manage, had something & NA & $2(0)$ & & NA & $1(1)$ & NA & $1(0.3)$ & \\
\hline Felt unwell & $88(15)$ & $39(7)$ & $8(4$ to 11$)$ & $30(12)$ & $17(7)$ & $58(16)$ & $22(7)$ & 0.33 \\
\hline \multicolumn{9}{|c|}{ Adverse effects rated as moderate or severe: } \\
\hline Abdominal pain or cramps & $56(9)$ & $59(10)$ & $-1(-5$ to 2$)$ & $23(9)$ & $21(9)$ & $33(9)$ & $38(12)$ & 0.44 \\
\hline Nausea or vomiting & $16(3)$ & $12(2)$ & $1(-1$ to 2$)$ & $7(3)$ & $8(3)$ & $9(3)$ & $4(1)$ & 0.25 \\
\hline Faintness or dizziness & $22(4)$ & $14(3)$ & $1(-1$ to 3$)$ & $10(4)$ & $9(4)$ & $12(3)$ & $5(2)$ & 0.30 \\
\hline Wind & $24(4)$ & $56(10)$ & $-6(-9$ to -3$)$ & $13(5)$ & $25(11)$ & $11(3)$ & $31(9)$ & 0.38 \\
\hline Bottom soreness $\dagger$ & $82(14)$ & $50(9)$ & 5 (1 to 8$)$ & $27(11)$ & $21(9)$ & $55(15)$ & $29(9)$ & 0.32 \\
\hline Incontinence & $8(1)$ & $28(5)$ & $-4(-6$ to -2$)$ & $6(2)$ & $12(5)$ & $2(1)$ & $16(5)$ & 0.10 \\
\hline Sleep disturbance & $6(1)$ & $74(13)$ & $-12(-15$ to -9$)$ & $3(1)$ & $36(15)$ & $3(1)$ & $38(12)$ & 0.94 \\
\hline Willing to use same preparation again & $543(89)$ & $501(89)$ & $0(-3$ to 5$)$ & $227(92)$ & $213(90)$ & $316(88)$ & $288(88)$ & 0.57 \\
\hline
\end{tabular}

* Significance test for homogeneity of effect between Welwyn Garden City and Leicester.

tIncludes pain or irritation in any of the following areas: rectum, anal canal, anus, skin around anus or buttock. 
Table 3 Numbers (percentages) of participants reporting adverse effects as moderate or severe on morning after test

\begin{tabular}{|c|c|c|c|c|c|c|c|c|}
\hline & \multicolumn{3}{|c|}{ Total } & \multicolumn{2}{|c|}{ Welwyn Garden City } & \multicolumn{2}{|c|}{ Leicester } & \multirow[b]{2}{*}{ P value } \\
\hline & Enema & Picolax & Difference in \% $(95 \% \mathrm{Cl})$ & Enema & Picolax & Enema & Picolax & \\
\hline Used allocated preparation & 607 & 566 & & 247 & 237 & 360 & 329 & \\
\hline Sent questionnaire† & 514 & 470 & & 154 & 141 & 360 & 329 & \\
\hline Responded to questionnaire & $490(95)$ & $435(93)$ & & $149(97)$ & $139(99)$ & $341(95)$ & $296(90)$ & \\
\hline Abdominal pain & $62(13)$ & $68(16)$ & $-3(-7$ to 2$)$ & $27(18)$ & $31(22)$ & $35(10)$ & $37(13)$ & 0.92 \\
\hline Nausea or vomiting & $3(1)$ & $6(1)$ & $0(-2$ to 1$)$ & $1(1)$ & $2(1)$ & $2(1)$ & $4(1)$ & 0.96 \\
\hline Faint or dizzy & $8(2)$ & $14(3)$ & $-2(-4$ to 0$)$ & $6(4)$ & $3(2)$ & $2(1)$ & $11(4)$ & 0.02 \\
\hline Wind & $157(32)$ & $146(34)$ & $-2(-8$ to 5$)$ & $72(48)$ & $71(51)$ & $85(25)$ & $75(25)$ & 0.77 \\
\hline Bottom soreness§ & $53(11)$ & $26(6)$ & 5 (1 to 8$)$ & $14(9)$ & $5(4)$ & $39(11)$ & $21(7)$ & 0.42 \\
\hline Incontinence & $28(6)$ & $21(5)$ & $1(-2$ to 4$)$ & $8(5)$ & $4(3)$ & $20(6)$ & $17(6)$ & 0.38 \\
\hline Sleep disturbance & $29(6)$ & $26(6)$ & $0(-3$ to 3$)$ & $5(3)$ & $1(1)$ & $24(7)$ & $25(8)$ & 0.12 \\
\hline Pain during test & $70(14)$ & $72(17)$ & $-2(-7$ to 2$)$ & $31(21)$ & $27(19)$ & $39(11)$ & $45(15)$ & 0.27 \\
\hline
\end{tabular}

*Significance test for homogeneity of effect between Welwyn Garden City and Leicester.

†Questionnaires were sent out in only the later stages of the study in Welwyn Garden City.

$\ddagger$ Abdominal pain and wind were reported significantly more often in Welwyn Garden City than Leicester because in Leicester carbon dioxide rather than air was used for insufflating the bowel. ${ }^{6}$

§Includes pain or irritation in any of the following areas: rectum, anal canal, anus, skin around anus or buttock.

Our study in two populations has shown that a single phosphate enema, self administered around one hour before leaving home for the examination, is more acceptable and effective than a single sachet of Picolax taken the day before, with dietary restriction until after the procedure. The greater acceptability of the enema was shown by higher attendance rates in both centres and higher compliance with the use of the preparation at home as intended. These findings contradict those of Lund et al in Nottingham, who compared home and hospital administered enemas in symptomatic patients and found that almost half of those randomised to receive an enema at home refused to use it at home and were given it at the hospital (the equivalent proportion in our study was $7 \%$ in both centres). ${ }^{9} \mathrm{We}$ did not compare hospital administered enema since, like many endoscopy units, our units did not have the facilities for all participants to be offered this option. Reasons cited in the Nottingham study for refusal to use the enema at home were: seemed too difficult (34\% of total offered home enema), fear of mess (14\%), and lack of understanding of instructions (4\%). In our study, $15 \%$ of those who attended after using the enema at home found it moderately difficult to use and $2 \%$ found it very difficult or needed to have help. Nevertheless, these participants had still used the preparation. Our success may have resulted from the carefully constructed information leaflet that was supplied and the availability of nursing support through a telephone advice line when required.

\section{Adverse effects}

More people reported feeling unwell immediately after using the enema, possibly because the enema acts within minutes whereas Picolax can take several hours to work. Overall, however, Picolax caused more adverse effects than the enema. The most important disadvantages of Picolax are, firstly, that it requires dietary

Table 4 Endoscopists' assessment of quality of bowel preparation. Values are numbers (percentages) of participants unless stated otherwise

\begin{tabular}{|c|c|c|c|c|c|c|c|c|}
\hline & \multicolumn{3}{|c|}{ Total } & \multicolumn{2}{|c|}{ Welwyn Garden City } & \multicolumn{2}{|c|}{ Leicester } & \multirow[b]{2}{*}{$P$ value ${ }^{*}$} \\
\hline & Enema & Picolax & Difference in $\%(95 \% \mathrm{CI})$ & Enema & Picolax & Enema & Picolax & \\
\hline No who used preparation at home & 635 & 584 & & 260 & 241 & 375 & 343 & \\
\hline Complete examinations (reaching descending colon or beyond) & $527(83)$ & $445(76)$ & $7(2$ to 11$)$ & $226(87)$ & $192(80)$ & $301(80)$ & $253(74)$ & 0.53 \\
\hline Incomplete examinations due to poor bowel preparation & $47(7)$ & $59(10)$ & $-3(-6$ to 0$)$ & $19(7)$ & $21(9)$ & $28(7)$ & $38(11)$ & 0.56 \\
\hline \multicolumn{9}{|l|}{ Quality of bowel preparation: } \\
\hline Excellent & $359(57)$ & $229(39)$ & \multirow{4}{*}{$\mathrm{P}<0.001$ for trend } & $115(44)$ & $71(29)$ & $244(65)$ & $158(46)$ & \\
\hline Good & $122(19)$ & $153(26)$ & & $82(32)$ & $92(38)$ & $40(11)$ & $61(18)$ & \\
\hline Poor & $62(10)$ & $92(16)$ & & $17(7)$ & $22(9)$ & $45(12)$ & $70(20)$ & \\
\hline Not recorded & $27(4)$ & $14(2)$ & & $13(5)$ & $4(2)$ & $14(4)$ & $10(3)$ & \\
\hline \multicolumn{9}{|l|}{ Mean \% mucosa visible† (No of patients) at: } \\
\hline $0-15 \mathrm{~cm}$ & $95(593)$ & $91(552)$ & $4(3$ to 6$)$ & $94(244)$ & $92(229)$ & $96(349)$ & $91(323)$ & \\
\hline $16-30 \mathrm{~cm}$ & $92(591)$ & $87(553)$ & 5 (3 to 8$)$ & $91(244)$ & $87(230)$ & $93(347)$ & $87(323)$ & \\
\hline $31-45 \mathrm{~cm}$ & $88(509)$ & $82(469)$ & $6(2$ to 10$)$ & $87(230)$ & $82(210)$ & $89(279)$ & $82(259)$ & \\
\hline $46-60 \mathrm{~cm}$ & $70(136)$ & $60(138)$ & $10(-1$ to 20$)$ & $70(57)$ & $72(63)$ & $70(79)$ & $51(75)$ & \\
\hline \multicolumn{9}{|l|}{ Yield at flexible sigmoidoscopy: } \\
\hline Polyps & $145(23)$ & $122(21)$ & $2(-3$ to 7$)$ & $55(21)$ & $52(22)$ & $90(24)$ & $70(20)$ & 0.42 \\
\hline Adenoma & $57(9)$ & $55(9)$ & $0(-4$ to 3$)$ & $27(10)$ & $27(11)$ & $30(8)$ & $28(8)$ & 0.88 \\
\hline Cancer & $5(1)$ & $2(0)$ & $0.5(-0.4$ to 1.3$)$ & $3(1)$ & $1(0)$ & $2(1)$ & $1(0)$ & 0.80 \\
\hline Total No of polyps & 206 & 188 & $0.5(0.5$ to 0.6$) \S$ & 84 & 79 & 122 & 109 & \\
\hline
\end{tabular}

*Significance test for homogeneity of bowel preparation effect between Welwyn Garden City and Leicester.

tIncludes examinations in which the scope was advanced to beyond the section of the bowel assessed unless the examination was terminated because of poor bowel preparation, in which case the $\%$ visible was set at $0 \%$ for subsequent sections.

¥Excluding examinations stopped due to inadequate bowel preparation and participants in whom polyps or cancer were detected.

$\S$ Binomial test for equality in two arms. 


\section{What is already known on this topic}

Screening by flexible sigmoidoscopy requires good bowel preparation

Staff and toilet facilities in endoscopy units are insufficient to cope with the large numbers of people seen in a screening programme

In the United States enemas are often self administered at home, but the acceptability of this approach in Britain is unknown

\section{What this study adds}

In asymptomatic people being screened by flexible sigmoidoscopy the compliance rates were higher among those randomised to home administration of enema than those receiving oral laxative

The enema group experienced fewer side effects and bowel preparation was better potentially embarrassing side effect, perhaps a pad should be offered to all subjects after the procedure.

Based on the results of this study, we believe that a single, self administered enema is probably the best available preparation for flexible sigmoidoscopy screening, and we have used this regimen in the UK flexible sigmoidoscopy screening trial, in which 40000 people aged 55-64 are being screened in 13 centres. The instructions used in this study have been incorporated into an illustrated booklet, and high compliance rates have been achieved.

We thank all the general practitioners and patients who participated; J F Mayberry, S Daniels, A Read, A C B Wicks, H Mackay, Y Rees, M J Kelly, J Lawson, S Burgin, and the endoscopy and day care nurses at Leicester General Hospital; Jennifer Pankhurst, Tracey Lewis, Kay Allwright, Dawn Webb, Pam Rogers, and the endoscopy nursing staff of the Queen's Wing, Queen Elizabeth II Hospital, Welwyn Garden City; Lin Squires, Steve Edmeades (Bioinformatics Unit), J Hickman, and P Sasieni (Mathematics, Statistics, and Epidemiology Unit), Imperial Cancer Research Fund; and Sheri Edwards, C B Williams, and I Talbot at St Mark's Hospital.

Contributors: WSA, AH, PM, RA, RE, JW, SS, JC, AS, and JMAN developed the study protocol. WSA, CB, and CFC analysed the data. WSA and CFC wrote the paper. All authors commented on the drafts. WSA is the guarantor for the paper.

Funding: The studies were funded by the Imperial Cancer Research Fund, the Medical Research Council, NHS Research and Development, and KeyMed.

Competing interests: Pharmax paid WSA a speaker's fee and contributed to the costs of meetings between trial investigators in the UK flexible sigmoidoscopy trial.

1 Atkin WS, Cuzick J, Northover JMA, Whynes DK. Prevention of colorectal cancer by once-only sigmoidoscopy. Lancet 1993;341:736-40.

2 Dakkak M, Aziz K, Bennett JR. Short report: comparison of two orally administered bowel preparations for colonoscopy-polyethylene glycol and sodium picosulphate. Aliment Pharmacol Ther 1992;6:513-9.

3 Weiss BD, Watkins S. Bowel preparation for flexible sigmoidoscopy. J Fam Pract 1985;21:285-7.

4 Verne J, Aubrey R, Love S, Talbot I, Northover J. Population based randomised study of uptake and yield of screening for flexible sigmoidoscopy compared with screening by faecal occult blood testing. BMJ 1998:317:182-5.

5 Preston K, Peluso F, Goldner F. Optimal bowel preparation for flexible sigmoidoscopy - are two enemas better than one? Gastrointest Endosc 1994;40:474-6.

6 Atkin WS, Hart A, Edwards R, McIntyre P, Aubrey R, Wardle J, et al. Uptake, yield of neoplasia and adverse effects of flexible sigmoidoscopy. Gut 1998;42:560-5.

7 Osgard E, Jackson J, Strong J. A randomized trial comparing three methods of bowel preparation for flexible sigmoidoscopy. Am J Gastroenterol 1998:93:1126-30.

8 Senapati A, Thomson MR. Domiciliary enemas for outpatient sigmoidoscopy. Gut 1992;33:S53.

9 Lund J, Buckley D, Bennett D, Maxwell-Armstrong C, Smith A, Tierney G, et al. A randomised trial of hospital versus home administered enemas for flexible sigmoidoscopy. BMJ 1998;317:1201.

10 Pietsch JB, Shizgal HM, Meakins JL. Injury by hypertonic phosphate enema. Can Med Assoc J 1977;1169-70.

11 Meisel J, Bergman D, Graney D, Saunders D, Rubin C. Human rectal mucosa: proctoscopic and morphological changes caused by laxatives. Gastroenterology 1977;72:1274-9.

12 Hixson L. Colorectal ulcers associated with sodium phosphate catharsis. Gastrointest Endosc 1995;42:101-2.

13 Thomas WM, Jackson J, Lamont GL, Hardcastle JD. Early incontinence following flexible sigmoidoscopy. Br J Surg 1990;77:887.

(Accepted 16 March 2000)

before reduced the need to repeat the proceduret $12 \%$ with two enemas to $4 \%$ in one study, ${ }^{7}$ although $16 \%$ complained of severe diarrhoea compared with only $6 \%$ receiving the enema alone. Administration of the enema in the unit by a nurse is not ideal, even if logistically feasible, since Thomas et al have reported that around $30 \%$ of people given an enema in the unit around 30 minutes before the procedure suffer incontinence of fluid or faeces on the journey home. ${ }^{13}$ Only $6 \%$ of our participants who used the enema complained of incontinence after sigmoidoscopy, a similar proportion to those using Picolax (5\%) (table 3). However, in view of the unacceptability of this

\section{Endpiece \\ A wise physician \\ Remedies often make disease worse.... It takes a wise doctor to know when not to prescribe. \\ Baltasar Gacian, 1601-58, The Art of Wordly Wisdom}

Submitted by T O Cheng, professor of medicine, Washington DC 


\title{
Commentary: participants should have been told they were being randomised
}

\author{
Henrik R Wulff
}

My personal "ethics meter" did not react very strongly, but it did react, when I read that the participants in this screening project had been randomised to two different cleansing procedures without their informed consent. The study was timely and the consequences for the patients were very small. However, informed consent is the rule, and violation of this rule must always be extremely well argued. The authors state that consent to randomisation was considered unnecessary as both methods were in routine use. This argument, taken in isolation, is certainly not valid. In some malignant diseases different chemotherapies are in current use, but that does not justify a randomised comparison of these regimens without the patients' consent.

My "ethics meter" would have reacted less if the authors had explained the ethical dilemma and argued their case in some detail. They should have made it clear that they had balanced the participants' right to self determination against the difficulty of securing informed written consent from 1400 people and the fact that the participants were exposed to no risk and no major inconvenience. However, many will still say that the right to self determination (the principle of autonomy) is so fundamental that it cannot be outbalanced by these other factors, and some critics of clinical research may even feel that a study such as this one justifies their distrust in the research activities of the medical profession.

What should the investigators have done? I think that they should have trusted the willingness of these people, who had already consented to take part in the screening project, to provide additional information for the benefit of future patients. The investigators should have explained openly by letter what it was all about and that randomisation was the only way to find out which cleansing procedure was best. If nothing was concealed from those who took part, the investigators could in this case, I believe, have refrained from the formality of written consent. Randomised trials are needed, and the investigators also missed a chance to propagate that message in the general population.

I have considered only the ethics of the study and not its legal aspects. In Denmark it would have been illegal without the participants' explicit consent.

Competing interests: None declared.

\section{Commentary: opportunity for patient partnership was lost}

Marie Taylor

Over the past seven years PASS Direct, a generic advocacy service in Dumfries and Galloway, has helped over 2000 patients in their dealings with the NHS. Many of these people have come to us with the complaint that they were not informed about the risks, side effects, or possible outcomes of procedures, surgery, or treatment. In all of these cases the reason for information being withheld has been an assumption on behalf of clinicians that the patient did not need to know; it was such a small risk, it wasn't worth mentioning; the patient would only worry; "I (the clinician) know best"; or the patient would not understand because he or she was not a doctor. Some of the patients were very angry that assumptions had been made about their ability to make choices for themselves, and all resolved to be better prepared in future.

The study by Atkin et al raises various issues about informing patients. It is my understanding that everyone who agreed to be screened was given an information leaflet describing the two methods. Therefore no one was uninformed that two methods were available. The issue seems to be that patients were sent a preparation rather than being asked which one they would like to try. However, having received a preparation they were given a choice of changing this or receiving assistance. I feel strongly that the intelligence of patients should never be underestimated and that in this case there was a lost opportunity to work in partnership with them.
As both preparations are widely used, and if we accept the fact that patients do not usually get either a choice or the support the patients in the trial received, the ethical issue, in my opinion, does not arise. When patients are kept well informed and given support, they are quite capable of asking questions. It is only when they are overly anxious, intimidated, or patronised that they lose the confidence to take part in what should be a shared decision making process.

The participants in this study seem to have been treated in a non-patronising manner and supported sensitively during the whole process. This in turn seems to have empowered them to ask for help or more information when they needed it. If, however, the participants had been suspected to have colorectal cancer, the situation would have been different. In these circumstances the patients would already have been disempowered and lacking in confidence and may have felt unable to ask questions. It would have been more appropriate in these circumstances to have given a straightforward choice after explaining both preparations.

In my experience as a patient's advocate, any information on procedures which can help people make choices should always be published. This is a lay person's perspective and may seem a little naive. However, had I been involved in this trial I would not feel that any code of ethics had been ignored, rather that the trial could have been a shared partnership one.

Competing interests: None declared.

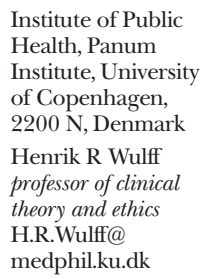

medphil.ku.dk
PASS Direct, Dumfries DG1 2AT Marie Taylor manager 